\title{
PERIODS OF HOLOMORPHIC MAPS ON COMPACT RIEMANN SURFACES AND PRODUCT OF SPHERES
}

\author{
JAUME LLIBRE ${ }^{1}$ AND VÍCTOR F. SIRVENT ${ }^{2}$ \\ Dedicated to O.M. Sharkovsky in his 82 nd birthday.
}

\begin{abstract}
In this article we consider non-constant holomorphic maps on Riemann surfaces and product of Riemann spheres, we give conditions on the maps in order that they have arbitrary large prime numbers as periods. We use Lefschetz fixed point theory and in particular we compute the Lefschetz numbers of period $m$ for large $m$ 's.
\end{abstract}

\section{INTRODUCTION}

In the theory of the dynamical systems and mainly in the study of the iteration of self-maps on a topological manifold $X$, the periodic orbits play an important role. More precisely, let $f: X \rightarrow X$ be a continuous map, a point $x \in X$ is periodic of period $k \in \mathbb{N}$ if $f^{k}(x)=x$ and $f^{j}(x) \neq x$ for $j=1, \ldots, k-1$. The set $\left\{x, f(x), \ldots, f^{k-1}(x)\right\}$ is the periodic orbit of the periodic point $x$ of period $k$. If $k=1$ then the periodic point $x$ is called a fixed point. We shall denote by $\operatorname{Per}(f)$ the set of periods of the map $f$. A natural question is to ask for all the possible periods that the map $f$ can exhibit. In some situations the knowledge of some possible periods of the map gives understanding of some global properties of the dynamics of the map, as in the case for continuous self-maps on the interval. If a continuous map on the interval has a periodic orbit of period three, then the map has orbits of all possible periods $(c f .[8,12])$.

The differential topological methods are very useful for understanding the periodic structure of continuous self-maps on manifolds on dimensions greater than 1 , because the topology of the manifold plays an important role, in particular we use the Lefschetz fixed point theory.

Date: September 6, 2019.

2010 Mathematics Subject Classification. 37C25,37C30,37E15.

Key words and phrases. Lefschetz numbers, holomorphic maps, periodic point, product of spheres, torus. 
In this article we study the periodic structure of non-constant holomorphic maps on Riemann surfaces and product of Riemann spheres, in particular we give conditions on homology such that the maps have arbitrary large prime numbers as periods; the corresponding results are Theorems 4, 5 and Corollary 8. In section 2 we consider the self-maps on Riemann surfaces and in section 3 the maps on product of Riemann spheres.

Let $X$ be an $n$-dimensional topological manifold and $f$ a continuous self-map on $X$. The map $f$ induces a homomorphism on the $k$-th rational homology group of $X$ for $0 \leq k \leq n$, i.e. $f_{* k}: H_{k}(X, \mathbb{Q}) \rightarrow$ $H_{k}(X, \mathbb{Q})$. The $H_{k}(X, \mathbb{Q})$ is a finite dimensional vector space over $\mathbb{Q}$ and $f_{* k}$ is a linear map whose matrix has integer entries.

The Lefschetz number of $f$ is defined as

$$
L(f):=\sum_{k=0}^{n}(-1)^{k} \operatorname{trace}\left(f_{* k}\right) .
$$

The Lefschetz Fixed Point Theorem states that if $L(f) \neq 0$ then $f$ has a fixed point ( $c f .[3]$ or [9]).

The Lefschetz numbers of period $m$, introduced in [4] and [10], are defined by

$$
\ell\left(f^{m}\right):=\sum_{r \mid m} \mu(r) L\left(f^{m / r}\right),
$$

where the sum is taken over all divisors $r$ of $m$ and $\mu$ is the Möbius function defined by

$$
\mu(m):= \begin{cases}1 & \text { if } m=1 ; \\ 0 & \text { if there is a } k \text { such that } k^{2} \text { divides } m ; \\ (-1)^{s} & \text { if } m=p_{1} \cdots p_{s} \text { with } p_{i} \text { distinct primes. }\end{cases}
$$

By the Möbius inversion formula

$$
L\left(f^{m}\right)=\sum_{r \mid m} \ell\left(f^{r}\right) .
$$

Observe that $L\left(f^{m}\right)$ and $\ell\left(f^{m}\right)$ are integer numbers for all $m$.

The Lefschetz zeta function of $f$ is defined as

$$
\zeta_{f}(t)=\exp \left(\sum_{m \geq 1} \frac{L\left(f^{m}\right)}{m} t^{m}\right)
$$

This function keeps the information of the Lefschetz number for all the iterates of $f$, so this function gives information about the set of periods 
of $f$. This function is rational, moreover its expression is

$$
\zeta_{f}(t)=\prod_{k=0}^{n} \operatorname{det}\left(I d_{* k}-t f_{* k}\right)^{(-1)^{k+1}},
$$

where $n=\operatorname{dim} M, n_{k}=\operatorname{dim} H_{k}(M, \mathbb{Q}), I d:=I d_{* k}$ is the identity map on $H_{k}(M, \mathbb{Q})$, and by convention $\operatorname{det}\left(I d_{* k}-t f_{* k}\right)=1$ if $n_{k}=0$ (cf. [6]).

Using formal computations we can write the Lesfchetz zeta function as a formal infinite product that involves the $\ell\left(f^{d}\right)$, for details see [1]:

$$
\zeta_{f}(t)=\exp \left(\sum_{m \geq 1} \frac{L\left(f^{m}\right)}{m} t^{m}\right)=\prod_{d \geq 1}\left(1-t^{d}\right)^{-\frac{\ell\left(f^{d}\right)}{d}} .
$$

The following result characterizes when the product in the identity (4) has a finite number of nontrivial factors, i.e. when $\ell\left(f^{d}\right) \neq 0$ for only finitely many $m$.

Theorem 1. Let $X$ be an $n$-dimensional topological manifold and $f$ a continuous self-map on $X$. The zeros and poles of the Lefschetz zeta function of $f$ are roots of unity if and only if $\ell\left(f^{m}\right) \neq 0$ for only finitely many $m$.

Proof. "The only if part". Let $m_{1}, \ldots, m_{k}$ be the only values of $m$ such that $\ell\left(f^{m}\right) \neq 0$. Accoding to $(4)$,

$$
\zeta_{f}(t)=\left(1-t^{m_{1}}\right)^{-\ell\left(f^{m_{1}}\right) / m_{1}} \cdots\left(1-t^{m_{k}}\right)^{-\ell\left(f^{m_{k}}\right) / m_{k}} .
$$

Therefore the roots and poles of $\zeta_{f}(t)$ are roots of unity.

"The if part". Since $\zeta_{f}(t)$ is a rational function it has only a finite number of poles and zeros, i.e. let $\omega_{1}, \ldots, \omega_{l}$ be such zeros and poles. So they are of the form $\omega_{j}=e^{2 \pi i r_{j} / k_{j}}$, for some positive integer $k_{j}$ and $0 \leq r_{j} \leq k_{j}-1$. According to Gauss's lemma the minimal polynomial of $\omega_{j}$ over the integers is the $k_{j}$-th cyclotomic polynomial, i.e. $\Phi_{k_{j}}(t)$. Hence $\Phi_{k_{1}}, \ldots \Phi_{k_{l}}$ are factors either in the numerator or denominator of $\zeta_{f}(t)$. By basic properties of the cyclotomic polynomials ( $\left.c f .[7]\right)$ :

$$
\Phi_{k_{j}}(t)=\prod_{d \mid k_{j}}\left(1-t^{d}\right)^{\mu\left(k_{j} / d\right)} .
$$

Therefore there exist non-zero integers $c_{i}$, with $1 \leq i \leq s$, for some $s$, such that

$$
\zeta_{f}(t)=\prod_{i=1}^{s}\left(1-t^{d_{i}}\right)^{c_{i}}
$$

where $d_{i}$ is a divisor of the least common multiple of $k_{1} \cdots k_{l}$. 
The relationship between the numbers $\ell\left(f^{m}\right)$ and the periodic structure for holomorphic maps was given in [5] in the following result.

Theorem 2 (Theorem A of [5]). Let $M$ be a compact complex manifold and $f: M \rightarrow M$ be a nonconstant holomorphic map. Then, there exists $M>0$ such that for all $p \in \mathbb{N}$ prime and $p>M, l\left(f^{p}\right) \neq 0$ if and only if $p \in \operatorname{Per}(f)$.

\section{Compact Riemann surfaces of genius $g$}

Let $X=M_{g}$ be a compact surface of genus $g$ endowed with a complex structure and $f: M_{g} \rightarrow M_{g}$ be a non-constant holomorphic map. The homology groups of $X$ with rational coefficients are:

$$
H_{0}\left(M_{g}, \mathbb{Q}\right)=H_{2}\left(M_{g}, \mathbb{Q}\right)=\mathbb{Q}, \quad H_{1}\left(M_{g}, \mathbb{Q}\right)=\underbrace{\mathbb{Q} \oplus \cdots \oplus \mathbb{Q}}_{2 g} .
$$

The identity (3) allows to write the Lefschetz zeta function of $f$ as

$$
\zeta_{f}(t)=\frac{p(t)}{(1-t)(1-D t)}
$$

where $D$ is the degree of $f$ and $p(t)=\operatorname{det}\left(\operatorname{Id}-t f_{* 1}\right)$, which is a polynomial of degree at most $2 g$.

Proposition 3. Let $f: M_{g} \rightarrow M_{g}$ be a continuous map. Then

(a) If $p(t)$ is not a product of cyclotomic polynomials, then $\ell\left(f^{m}\right) \neq$ 0 for infinitely many $m$.

(b) If $|D| \neq 0,1$ and $D^{-1}$ is not an eigenvalue of $f_{* 1}$ of multiplicity 1 , then $\ell\left(f^{m}\right) \neq 0$ for infinitely many $m$.

Proof. For continuous self-maps on $M_{g}$ an expression of its Lefschetz zeta function is given in (5), which is written in irreducible form, i.e. $1-t$ and $1-D t$ are not factors of $p(t)$. If $p(t)$ is not a product of cyclotomic polynomials then $\zeta_{f}(t)$ has zeros which are not roots of unity, according to Theorem 1 , there are infinitely many $m$ 's such that $\ell\left(f^{m}\right) \neq 0$.

If $D \neq 0$ and $D^{-1}$ is not an eigenvalue of $f_{* 1}$ of multiplicity 1 , then $D^{-1}$ is a root or pole of $\zeta_{f}(t)$, because $p(t)=\operatorname{det}\left(I d-t f_{* 1}\right)$. So if $D \neq-1,1$ since $D$ is an integer, we have that $\zeta_{f}(t)$ has a root or pole which is not a root of unity. Therefore $\ell\left(f^{m}\right) \neq 0$ for infinitely many m's.

From Proposition 3 and Theorem 2 it yields the following result. 
Theorem 4. Let $M_{g}$ be an orientable surface of genus $g$ endowed with a complex structure and $f: M_{g} \rightarrow M_{g}$ be a nonconstant holomorphic map such that its Leftschetz zeta function is given in (5).

(a) If $p(t)$ is not a product of cyclotomic polynomials.

(b) If $|D| \neq 0,1$ and $D^{-1}$ is not an eigenvalue of $f_{* 1}$ of multiplicity 1.

Then there exists an integer $N>0$ such that for all prime $p>N$, $p$ is a period of the map $f$.

Theorem 5. Let $M_{g}$ be an orientable surface of genus $g$ endowed with a complex structure and $f: M_{g} \rightarrow M_{g}$ be a nonconstant holomorphic map. Let $\lambda_{1}, \ldots \lambda_{2 g}$ be the eigenvalues of $f_{* 1}$ and $D$ the degree of $f$. If any of these conditions hold

(a) $|D|>\max \left\{1,\left|\lambda_{1}\right|, \ldots,\left|\lambda_{2 g}\right|\right\}$.

(b) There exists $\left|\lambda_{j}\right|>\max \left\{1,|D|,\left|\lambda_{i}\right|: i \neq j\right\}$.

(c) There exists $i_{1}, \ldots, i_{k}$ such that $\lambda_{i_{1}}=\cdots=\lambda_{i_{k}}$ and

$$
\left|\lambda_{i_{1}}\right|>\max \left\{1,|D|,\left|\lambda_{j}\right|: j \neq i_{1}, \ldots, i_{k}\right\} .
$$

(d) There exists $i_{1}, \ldots, i_{k}$ such that

$$
\left|\lambda_{i_{1}}\right|=\cdots=\left|\lambda_{i_{k}}\right|>\max \left\{1,|D|,\left|\lambda_{j}\right|: j \neq i_{1}, \ldots, i_{k}\right\} .
$$

Then there exists an integer $N>0$ such that for all prime $p>N, p$ is a period of the map $f$.

Proof. From the definition of the Lefschetz numbers (1) and for all $m \in \mathbb{N}$ we have

$$
L\left(f^{m}\right)=1-\left(\lambda_{1}^{m}+\cdots+\lambda_{2 g}^{m}\right)+D^{m} .
$$

For $p$ prime, according to (2)

$$
\begin{aligned}
\ell\left(f^{p}\right) & =L\left(f^{p}\right)-L(f) \\
& =\left(1-\left(\lambda_{1}^{p}+\cdots+\lambda_{2 g}^{p}\right)+D^{p}\right)-\left(1-\left(\lambda_{1}+\cdots+\lambda_{2 g}\right)+D\right) \\
& =\left(D^{p}-D\right)-\left(\lambda_{1}^{p}-\lambda_{1}\right)-\cdots-\left(\lambda_{2 g}^{p}-\lambda_{2 g}\right) .
\end{aligned}
$$

If conditions (a) or (b) are held, it can be easily checked that $\ell\left(f^{p}\right) \neq$ 0 for sufficiently large prime $p$. Hence, by Theorem 2 these prime numbers belong to Per(f).

If $\lambda_{i_{1}}=\cdots=\lambda_{i_{k}}$, the number $\ell\left(f^{p}\right)$ can be written as

$$
\ell\left(f^{p}\right)=-k\left(\lambda_{i_{1}}^{p}-\lambda_{i_{1}}\right)+\left(D^{p}-D\right)-\sum_{j \neq i_{1}, \ldots i_{k}}\left(\lambda_{j}^{p}-\lambda_{j}\right) .
$$


Hence

$$
\begin{aligned}
\left|\ell\left(f^{p}\right)\right| & \geq k\left|\lambda_{i_{1}}^{p}-\lambda_{i_{1}}\right|-\left|D^{p}-D\right|-\sum_{j \neq i_{1}, \ldots i_{k}}\left|\lambda_{j}^{p}-\lambda_{j}\right| \\
& =\left|\lambda_{i_{1}}^{p}-\lambda_{i_{1}}\right|\left(k-\frac{\left|D^{p}-D\right|}{\left|\lambda_{i_{1}}^{p}-\lambda_{i_{1}}\right|}-\sum_{j \neq i_{1}, \ldots i_{k}} \frac{\left|\lambda_{j}^{p}-\lambda_{j}\right|}{\left|\lambda_{i_{1}}^{p}-\lambda_{i_{1}}\right|}\right) .
\end{aligned}
$$

If $\left|\lambda_{i_{1}}\right|>\max \left\{1,|D|,\left|\lambda_{j}\right|: j \neq i_{1}, \ldots, i_{k}\right\}$, the ratios

$$
\frac{\left|D^{p}-D\right|}{\left|\lambda_{i_{1}}^{p}-\lambda_{i_{1}}\right|}, \quad \frac{\left|\lambda_{j}^{p}-\lambda_{j}\right|}{\left|\lambda_{i_{1}}^{p}-\lambda_{i_{1}}\right|}
$$

are arbitrary small for sufficiently large $p$. Hence $\left|\ell\left(f^{p}\right)\right|>0$ for large prime numbers $p$. So by Theorem 2, these numbers $p$ belong to $\operatorname{Per}(f)$. This proves statement (c).

$$
\begin{aligned}
\text { If }\left|\lambda_{i_{1}}\right| & =\cdots=\left|\lambda_{i_{k}}\right|>1 \text { then } \\
\qquad\left(f^{p}\right) \mid & \geq\left(\left|\lambda_{i_{1}}^{p}-\lambda_{i_{1}}+\cdots+\lambda_{i_{k}}^{p}-\lambda_{i_{k}}\right|\right)-\left|D^{p}-D\right|-\sum_{j \neq i_{1}, \ldots i_{k}}\left|\lambda_{j}^{p}-\lambda_{j}\right| \\
& =\left|\lambda_{i_{1}}\right|^{p-1}\left(\left(\lambda_{i_{1}}-1\right)+\cdots+\left(\lambda_{i_{k}}-1\right)\right)-\left|D^{p}-D\right|-\sum_{j \neq i_{1}, \ldots i_{k}}\left|\lambda_{j}^{p}-\lambda_{j}\right| \\
& \geq C_{1}\left|\lambda_{i_{1}}\right|^{p}-\left|D^{p}-D\right|-\sum_{j \neq i_{1}, \ldots i_{k}}\left|\lambda_{j}^{p}-\lambda_{j}\right|,
\end{aligned}
$$

for some constant $C_{1}>0$.

Hence

$$
\left.\ell\left(f^{p}\right)|\geq| \lambda_{i_{1}}\right|^{p}\left(C_{1}-\frac{\left|D^{p}-D\right|}{\left|\lambda_{i_{1}}\right|^{p}}-\sum_{j \neq i_{1}, \ldots i_{k}} \frac{\left|\lambda_{j}^{p}-\lambda_{j}\right|}{\left|\lambda_{i_{1}}\right|^{p}}\right) .
$$

If $\left|\lambda_{i_{1}}\right|>\max \left\{1,|D|,\left|\lambda_{j}\right|: j \neq i_{1}, \ldots, i_{k}\right\}$ then the ratios

$$
\frac{\left|D^{p}-D\right|}{\left|\lambda_{i_{1}}\right|^{p}}, \quad \frac{\left|\lambda_{j}^{p}-\lambda_{j}\right|}{\left|\lambda_{i_{1}}\right|^{p}}
$$

are small for arbitrary large $p$. Therefore we can conclude $\ell\left(f^{p}\right) \neq 0$, for large $p$. This proves statement $(\mathrm{d})$.

We note that the minimal periods of holomorphics maps on surfaces has been studied in [11]. 


\section{Product of Riemann spheres}

In this section we consider the product of Riemann spheres, which have a natural complex structure. We define $X(n):=\underbrace{\mathbb{S}^{2} \times \cdots \times \mathbb{S}^{2}}_{n \text {-times }}$.

Theorem 6. Let $f: X(n) \rightarrow X(n)$ be a continuous map. Let $\lambda_{1}, \cdots, \lambda_{n}$ be the eigenvalues of $f_{* 2}$.

(a) If $\left|\lambda_{i}\right|>1$ for $1 \leq i \leq n$, then $\ell\left(f^{p}\right) \neq 0$ for all prime number $p$.

(b) If $\lambda_{i}=-1$ for some $i$ then $\ell\left(f^{p}\right)=0$, for all odd prime numbers.

(c) If $\lambda_{i}=-1$ for some $i$ and $\lambda_{j} \neq 0$ for $1 \leq j \leq n$ then $\ell\left(f^{2}\right) \neq 0$.

Before proving Theorem 6 we present the following result that determines the value of the Lefschetz numbers for the maps on $X(n)$.

Theorem $7([2])$. Let $f: X(n) \rightarrow X(n)$ be a continuous map. Then, for all $m>0$,

$$
L\left(f^{m}\right)=\operatorname{det}\left(I d+f_{* 2}^{m}\right),
$$

where Id is the identity map on $\mathbb{Q}^{n}$. In particular,

$$
L\left(f^{m}\right)=(-1)^{n} C_{f_{* 2}^{m}}(-1),
$$

where $C_{h}(t):=\operatorname{det}(t I d-h)$ denotes the characteristic polynomial of $h$.

Proof of Theorem 6. Due to Theorem 7, we have

$$
L\left(f^{m}\right)=(-1)^{n}\left(1+\lambda_{1}^{m}\right) \cdots\left(1+\lambda_{n}^{m}\right) .
$$

If $p$ is a prime number then

(6) $\ell\left(f^{p}\right)=L\left(f^{p}\right)-L(f)$

$$
=(-1)^{n}\left(\left(1+\lambda_{1}^{p}\right) \cdots\left(1+\lambda_{n}^{p}\right)-\left(1+\lambda_{1}\right) \cdots\left(1+\lambda_{n}\right)\right) .
$$

If $\lambda_{i}>1$ for $1 \leq i \leq n$ then $\lambda_{i}^{p}>\lambda_{i}$ so $(-1)^{n} \ell\left(f^{p}\right)>0$ for every prime $p$.

If $\lambda_{i}<-1$ for $1 \leq i \leq n$ then $\lambda_{i}^{p}<\lambda_{i}$ for every prime $p>2$. If $n$ is even then $\ell\left(f^{p}\right)>0$ and if $n$ is odd $\ell\left(f^{p}\right)<0$. Similarly if $p=2$ then $(-1)^{n} \ell\left(f^{p}\right)>0$ for all $n$. This proves statement (a).

If $\lambda_{i}=-1$ for some $i$, then it follows from (7) that $\ell\left(f^{p}\right)=0$ for $p$ an odd prime. Moreover if $\lambda_{j} \neq 0$ for all $1 \leq j \leq n$ then, from (7) we have $\ell\left(f^{2}\right) \neq 0$. This proves statements (b) and (c), respectively. 
The following corollary is a consequence of Theorem 6 and Theorem 2.

Corollary 8. Let $f: X(n) \rightarrow X(n)$ be a non-constant holomorphic map. Let $\lambda_{1}, \cdots, \lambda_{n}$ be the eigenvalues of $f_{* 2}$. If $\left|\lambda_{i}\right|>1$, for $1 \leq i \leq n$ then there exists an integer $N$ such that all prime numbers $p>N$ are periods of $f$.

Theorem 9. Let $f: X(n) \rightarrow X(n)$ be a non-constant holomorphic map. If $f_{* 2}$ has an eigenvalue bigger than one in modulus and that it does not have root of unity as eigenvalues. Then there exists $N$ such that all prime numbers $p>N$ are periods of $f$.

Theorem 9 is a particular case of the following more general result proved in [13].

Theorem 10. Let $f: X(n) \rightarrow X(n)$ be a continuous map. We assume that $f_{* 2}$ has an eigenvalue bigger than one in modulus and that it does not have root of unity as eigenvalues. Then there exists $N$ such that $\ell\left(f^{m}\right) \neq 0$ for $m \geq N$.

\section{ACKNOWLEDGEMENTS}

The first author is partially supported by the Ministerio de Ciencia, Innovación y Universidades, Agencia Estatal de Investigación grants MTM2016-77278-P (FEDER), the Agència de Gestió d'Ajuts Universitaris i de Recerca grant 2017SGR1617, and the H2020 European Research Council grant MSCA-RISE-2017-777911.

\section{REFERENCES}

[1] I. K. Babenko and S. A. Bogatyi, The behaviour of the index of periodic points under iterations of a mapping, Math. USSR Izv., 38 (1992), 1-26.

[2] P. Berrizbeitia, M.J. GonzÁlez, and V.F. Sirvent, On the Lefschetz zeta function and the minimal sets of Lefschetz periods for Morse-Smale diffeomorphisms on products of $\ell$-spheres, Topology Appl. 235 (2018) 428-444.

[3] R.F. Brown, The Lefschetz fixed point theorem, Scott, Foresman and Company, Glenview, IL, 1971.

[4] A. Dold, Fixed point indices of iterated maps, Invent. math. 74 (1983), 419435.

[5] N. Fagella And J. Llibre, Periodic points of holomorphic maps via Lefschetz numbers, Trans. Amer. Math. Soc. 352 (2000), 4711-4730.

[6] J. Franks, Period doubling and the Lefschetz formula, Trans. Amer. Math. Soc. 287 (1985), 275-283.

[7] S. LAng, Algebra, Addison-Wesley, Boston, MA, 1971. 
[8] T.Y. Li and J. Yorke, Period three implies chaos, Amer. Math. Monthly 82 (1975), 985-992.

[9] S. LefsChetz, Intersections and transformations of complexes and manifolds, Trans. Amer. Math. Soc. 28 (1926), 1-49.

[10] J. LliBRE, Lefschetz numbers for periodic points, Contemporary Math., vol 152 (1993), 215-227, Amer. Math. Soc., Providence.

[11] J. Llibre And W. Marzantowicz, Periods of holomorphic maps on surfaces, Funtiones et Approximatio 40.2 (2009), 309-326.

[12] O.M. Sharkovsky, Co-existence of cycles of a continuous mapping of the line into itself (Russian), Ukrain. Mat. Zh. 16 (1964), 61-71.

[13] V.F. SiRvent A note on the periodic structure of transversal maps on the torus and the product of spheres, Pre-print (submitted).

${ }^{1}$ Departament de Matemàtiques. Universitat Autònoma de Barcelona, Bellaterra, 08193 Barcelona, Catalunya, Spain

Email address: jllibre@mat.uab.cat

2 Departamento de Matemáticas, Universidad Católica del Norte, Antofagasta, Chile

Email address: victor.sirvent@ucn.cl 